\title{
Usefulness of Mouth Guard in Active Sports
}

\author{
Jitender Batra*, Gyanander Attresh and Shanander Sambyal \\ Department of Oral \& Maxillofacial Surgery, Post Graduate Institute of Dental Sciences, Pt. B.D Sharma U.H.S, Rohtak, Haryana, India
}

\begin{abstract}
Mouth guard is a flexible appliance worn over the teeth by the persons engaged in contact sports. Mouth guards reduce the traumatic impacts and act by preventing the impact from reaching the oral region or by cushioning, absorbing, or distributing the impact forces. They can be of stock type; mouth formed; or custom fitted type. Mouth Guard helps to protect against chipped or broken teeth, root and bone damage, and tooth loss and safeguards against serious injuries such as jaw fracture, cerebral hemorrhage, concussion and neck injuries. Compulsory and regular use of a mouth guard is beneficial in both ways i.e. protection from injury and increase in sportsman efficiency in certain sports. This short communication also highlights the basic requirements and uses of mouth guards.
\end{abstract}

Keywords: Sports injuries; Prevention; Mouth guards

\section{Introduction}

Traumatic impacts provoke an acute delivery of energy that is released upon soft and hard tissues, resulting in laceration, contusion, or ablation of tissues. Mouth guards reduce the consequences of such impacts and act by preventing the impact from reaching the oral region or by cushioning, absorbing, or distributing the impact forces [1].

\section{Types}

Mouth guards are available in three types: (1) Stock mouth guards, which can be purchased in sporting goods and drug stores, come preformed and ready to wear; (2) Mouth-formed mouth guards which can be either a shell liner or a boil-and-bite kind; and (3) Custom-fitted mouth guards which are more expensive because they are made from a cast to precisely fit the teeth and provide the greatest degree of fit, comfort and protection [2].

\section{Ideal requirements}

1. They should enclose the maxillary teeth to the distal surface of the second molars.

2. Thickness should be $3 \mathrm{~mm}$ on the labial aspects, $2 \mathrm{~mm}$ on the occlusal aspect, and $1 \mathrm{~mm}$ on the palatal aspect.

3. The labial flange should extend to within $2 \mathrm{~mm}$ of the vestibular reflection.

4. The palatal flange should extend about $10 \mathrm{~mm}$ above the gingival margin.

5. The edge of the labial flange should be rounded in cross section whereas the palatal edge should be tapered.

6. When a maxillary guard is constructed it should be articulated against the matching mandibular model for optimum comfort [3].

\section{Uses}

Wearing a mouth guard is an important precaution for athletes of all ages and abilities [4];

- Helping to protect against chipped or broken teeth, root and bone damage, and tooth loss.

- Safeguard against serious injuries such as jaw fracture, cerebral hemorrhage, concussion and neck injuries by helping to avoid situations where the lower jaw jams into the upper jaw.

- By keeping soft tissue in the oral cavity away from the teeth, mouth guards help prevent cutting and bruising of the lips, tongue and cheeks, especially for athletes who wear orthodontic appliances.

- Other important advantage of using mouth guard is that they prevent development of Temporomandibular Joint (TMJ) Disorders mainly internal derangement. The basic theory being that the athlete, while lifing to his peak of performance, naturally clenches his jaw and thus loads the joint with excessive forces. This in long term results in development of internal derangement of TMJ i.e. malrelationship of the articular disc to the condylar head and the articular eminence. It can lead to the sequelae of pain, joint noises and restricted motion.

- Moreover, the mouth guard restrains the athlete from clenching thus redistributing that energy back to the athlete allowing him to lift more weight or perform more repetitions with the mouth guard in place. The mouth guard with power bites also provides an open airway for the athlete that he would not have through clenched teeth.

\section{Conclusion}

Compulsory and regular use of a mouth guard is beneficial in both ways i.e. protection from injury and increase in sportsman efficiency in certain sports.

\section{References}

1. Bourguignon C, Sigurdsson A (2009) Preventive strategies for traumatic dental injuries. Dent Clin North Am 53: 729-749.

2. Patrick DG, van Noort R, Found MS (2005) Scale of protection and the various types of sports mouth guard. Br J Sports Med. 39: 278-281.

3. Scott J, Burke FJT, Watts DC (1994) A review of dental injuries and the use of mouthguards in contact team sports. Br Dent J 176: 310-314.

4. Ranalli DN, Elderkin DL (2006) Oral health issues for adolescent athletes. Dent Clin North Am 50:119-137.

*Corresponding author: Jitender Batra, Department of Oral \& Maxillofacia Surgery, Post Graduate Institute of Dental Sciences, Pt. B.D Sharma U.H.S, Rohtak, Haryana, 124001, India, Tel: +918929956599; E-mail: dr.batrajatin@gmail.com

Received June 28, 2014; Accepted September 03, 2014; Published September 15,2014

Citation: Batra J, Attresh G, Sambyal S (2014) Usefulness of Mouth Guard in Active Sports. J Sports Med Doping Stud 4: 146. doi:10.4172/2161-0673.1000146

Copyright: ( 2014 Batra J, et al. This is an open-access article distributed under the terms of the Creative Commons Attribution License, which permits unrestricted use, distribution, and reproduction in any medium, provided the original author and source are credited. 